University of Nebraska - Lincoln

DigitalCommons@University of Nebraska - Lincoln

\title{
Dinosaur Tectonics: A Structural Analysis of Theropod Undertracks with a Reconstruction of Theropod Walking Dynamics
}

\author{
Ole Graversen \\ University of Copenhagen \\ Jesper Milán \\ University of Copenhagen \\ David B. Loope \\ University of Nebraska, Lincoln, dloope1@unl.edu
}

Follow this and additional works at: https://digitalcommons.unl.edu/geosciencefacpub

Part of the Earth Sciences Commons

Graversen, Ole; Milán, Jesper; and Loope, David B., "Dinosaur Tectonics: A Structural Analysis of Theropod Undertracks with a Reconstruction of Theropod Walking Dynamics" (2007). Papers in the Earth and Atmospheric Sciences. 213.

https://digitalcommons.unl.edu/geosciencefacpub/213

This Article is brought to you for free and open access by the Earth and Atmospheric Sciences, Department of at DigitalCommons@University of Nebraska - Lincoln. It has been accepted for inclusion in Papers in the Earth and Atmospheric Sciences by an authorized administrator of DigitalCommons@University of Nebraska - Lincoln. 


\title{
Dinosaur Tectonics: A Structural Analysis of Theropod Undertracks with a Reconstruction of Theropod Walking Dynamics
}

\author{
Ole Graversen, Jesper Milàn, and David B. Loope ${ }^{1}$ \\ Geology Section, Department of Geography and Geology, University of Copenhagen, Geocenter Copenhagen, \\ Øster Voldgade 10, DK-1350 Copenhagen K, Denmark \\ (e-mail: oleg@geol.ku.dk)
}

\begin{abstract}
A B S T R A C T
A dinosaur trackway in the Middle Jurassic eolian Entrada Sandstone of southern Utah, U.S.A., exposes three undertracks that we have modeled as isolated tectonic regimes showing the development of fold-thrust ramp systems induced by the dinosaur's feet. The faulted and folded sequence is comparable to crustal-scale tectonics associated with plate tectonics and foreland fold-thrust belts. A structural analysis of the dinosaur tracks shows the timing and direction of the forces exercised on the substrate by the animal's foot during the stride. Based on the structural analysis, we establish a scenario for foot movements and weight distribution in the feet. During the end of the weightbearing phase of the stride, the weight of the animal was transferred to the front of the digits, creating a rotated disk below the foot that was bounded by an extensional fault at the front and a thrust ramp toward the back. As the body accelerated, the foot was forced backward. The rotated disk was forced backward along a detachment fault that was bounded by lateral ramps. The interramp segment matches the width of the dinosaur's foot, which created an imbricate fan thrust system that extended to the far end of the undertrack. The total length of the tectonic disturbance created by the dinosaur is up to three times that of the original footprint. Early, near-surface cementation gave the substrate the rheological properties necessary for development of the observed structures.
\end{abstract}

\section{Introduction}

A footprint is a complex structure resulting from the dynamic contact between an animal and the properties of the substrate. During the ground contact, not only the actual tracking surface (sensu Fornós et al. 2002) but also the subjacent horizons are deformed as the pressure from the track maker's foot is transferred downward and radially outward into the sediment (Allen 1989, 1997; Gatesy 2003; Manning 2004; Milàn and Bromley 2006). This effect is best studied in tracks left in finely layered heterolithic sediments, where the different lithologies allow the sediment packages to be split along several subjacent horizons, exposing the stacked succession of undertracks that gradually become wider, lower in relief, and less detailed downward (Milàn and Bromley 2006).

During the time of contact between the animal's foot and the substrate, any simultaneous move2007.

Manuscript received December 29, 2006; accepted June 4,

${ }^{1}$ Department of Geosciences, University of Nebraska, Lincoln, Nebraska 68588-0340, U.S.A. ment of the foot may be captured by the sediment and recognized as a zone of deformation within and around the footprint (Brown 1999). Theropod tracks from the Late Triassic of Greenland have preserved exquisite skin impressions of a rough tubercular skin covering the sole of the theropod's digits. By careful study of the orientation of the striations left by the tubercular skin being dragged through the sediment, Gatesy (2001) concluded that a lateral flattening of the fleshy parts covering the digits occurred while weight was applied onto the track maker's foot, forcing the sediment outward.

Studies of vertical sections through theropod tracks from the Late Triassic (Milàn et al. 2004), Early Jurassic (Avanzini 1998), and Late Jurassic (Milàn et al. 2006) demonstrate that the movements of the track maker's feet during the contact with the ground are transmitted deep into the subjacent sediment layers and are recognizable in the undertracks. Study of sections cut through tracks and the subjacent sediments can further reveal differences in timing and distribution of the weight

[The Journal of Geology, 2007, volume 115, p. 641-654] @ 2007 by The University of Chicago. All rights reserved. 0022-1376/2007/11506-0003\$15.00 DOI: 10.1086/521608 

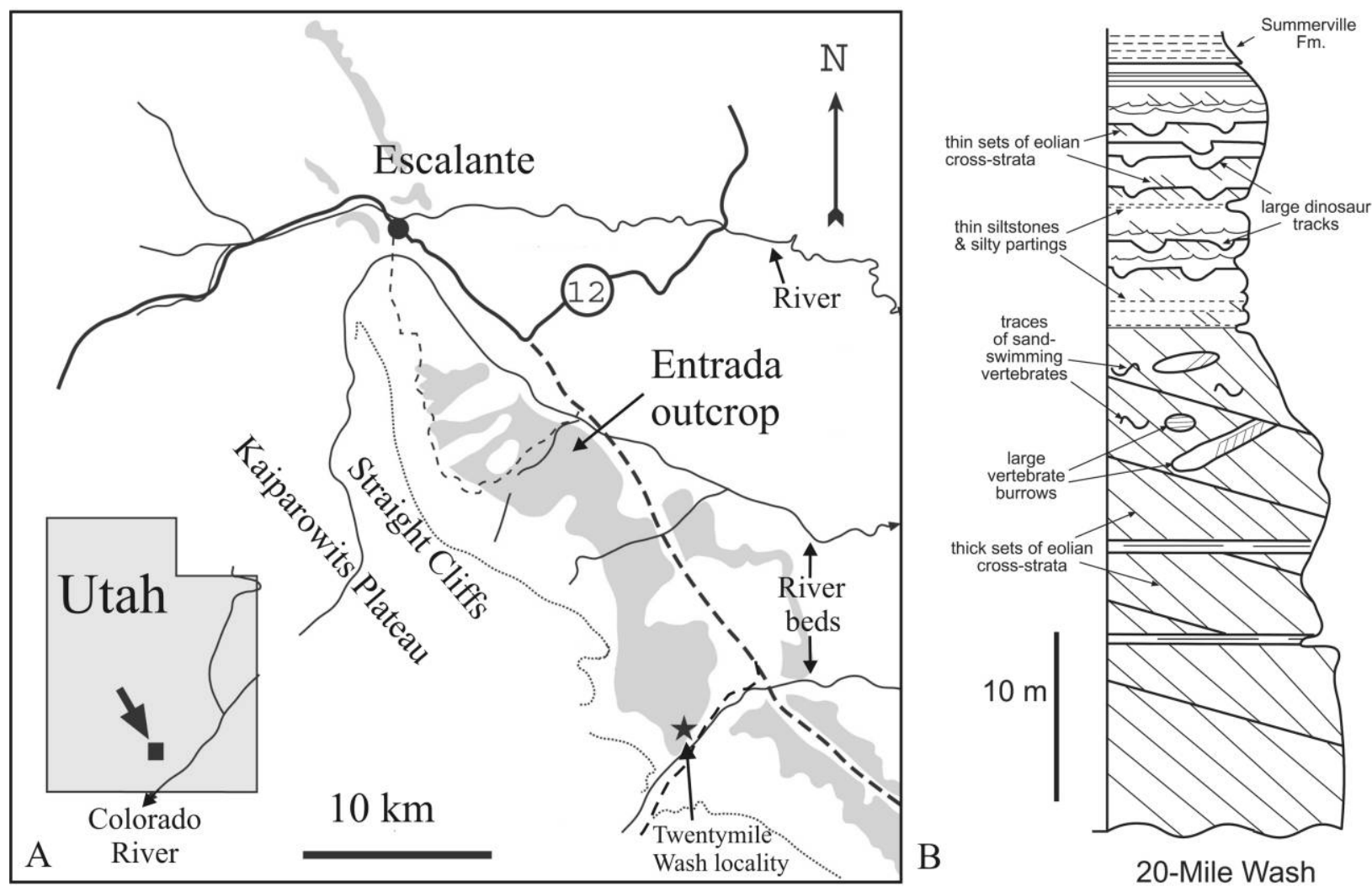

Figure 1. A, Location map. The Twentymile Wash dinosaur track site is located in the southern part of Utah, southeast of the town of Escalante $\left(37^{\circ} 33^{\prime} 05^{\prime \prime} \mathrm{N}, 111^{\circ} 25^{\prime} 24^{\prime \prime} \mathrm{W}\right)$, indicated by a star. Thick solid and dashed lines indicate roads, while thin dashed line indicates smaller trail and thin solid lines show riverbeds. Shaded areas indicate the present-day outcrops of the Entrada Sandstone. The front of the straight cliffs is indicated by a thin dotted line. $B$, Stratigraphic section of Entrada Sandstone exposed at Twentymile Wash locality. The large dinosaur tracks are found in the upper laminated part of the section.

load exercised on the different parts of the foot during the stride. This is evidenced by sideways-displaced rims of sediment around and below the imprint of the foot (Milàn et al. 2006).

The first aim of this study is to make a structural analysis of the faults and joints occurring in the sediment around eroded theropod undertracks from the Middle Jurassic Entrada Sandstone. The structural analysis will then form the basis for a reconstruction of theropod walking dynamics with special emphasis on the timing and shifting of the weight distribution on the feet during the stride.

\section{Study Area}

The footprints analyzed in this study are found in the Escalante Member, in the uppermost part of the Middle Jurassic Entrada Sandstone of the San Rafael Group (Thompson and Stokes 1970). The Entrada Sandstone forms a prominent, 65-68-m-thick, white to gray, cliff-forming unit along the northeastern margin of the Kaiparowits Plateau, at the Twentymile Wash locality, about $30 \mathrm{~km}$ southeast of the town of Escalante in southern Utah (fig. 1). Dinosaur tracks have previously been described from the Twentymile Wash locality (e.g., Foster et al. 2000; Breithaupt et al. 2004; Milàn and Loope 2007). The majority of the dinosaur footprints found in the Escalante Member are preserved within flat-bedded eolian sand sheets and interbedded thin sets of eolian cross-laminated strata that accumulated above a shallow water table (Loope and Simpson 1992). The tracks may have been formed during moist, pluvial periods, when the summer monsoon precipitation maintained a high water table in the dune field (Loope and Rowe 2003).

The dinosaur ichnofauna at the Twentymile Wash locality is dominated by theropod footprints ranging in length from 15 to $45 \mathrm{~cm}$, but footprints 

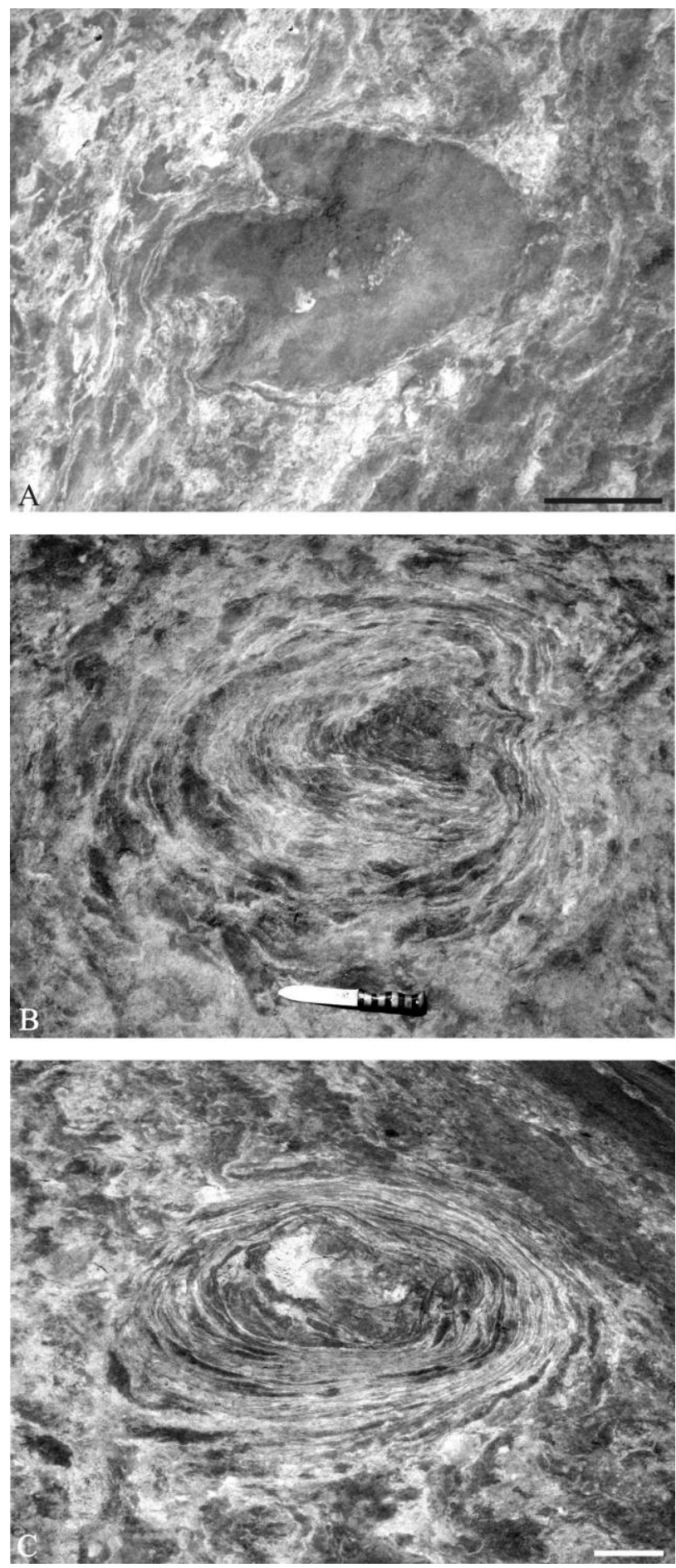

Figure 2. Theropod tracks at the Twentymile Wash locality exposed in different preservational styles and degrees of erosion. $A$, True track of a large theropod infilled with sand of a darker color. The shape and outline of the track are well defined, with clear impressions from the claws. Scale bar $=10 \mathrm{~cm}$. $B$, True track here eroded away, with the tridactyl shape only vaguely recognizable in the as small as $4 \mathrm{~cm}$ are occasionally encountered. The dinosaur tracks of the Entrada-Summerville transition zone can be traced in various outcrops in Utah over about $1000 \mathrm{~km}^{2}$ as part of the Moab Megatracksite (Lockley and Hunt 1995; Lockley 1997). The larger of the theropod footprints that are the subject of this study, have been assigned to the ichnogenus Megalosauripus and the smaller footprints to Therangospodus (Foster et al. 2000). In addition to the abundant footprints of theropods are two wide-gauge quadrupedal trackways assigned to the sauropod ichnogenus Brontopodus (Foster et al. 2000).

\section{Footprint Preservation}

The dinosaur tracks found at the Twentymile Wash locality are exposed in a wide range of preservational styles because of the present-day subaerial erosion of the sandstone. The tracks are exposed in a variety of random erosional cuts and as undertracks formed on the horizons subjacent to the original tracking surface. Many of the theropod tracks are preserved as true tracks (sensu Lockley 1991). True tracks reflect the original shape of the dinosaur's feet and can, if well preserved, reveal important details about the soft-part anatomy of the animal's feet. When the dinosaurs crossed the Twentymile Wash site, they not only deformed the surface but also folded and ruptured the subjacent layers of sand $20 \mathrm{~cm}$ below the track (Milàn and Loope 2007).

Many of the true tracks at the Twentymile Wash locality are infilled with darker sediment, which makes the casts of the footprints stand out in contrast with the lighter-colored background (fig. 2A). As a result of erosion, a majority of the tracks are preserved only as undertracks. A typical undertrack morphology at the Twentymile Wash locality is that of a vaguely defined undertrack, where it is still possible to identify the imprints of the three digits and a surrounding zone of deformation around the remains of the true track (fig. $2 B$ ). Where erosion has proceeded to such an extent that the true tracks are completely eroded away, a promi-

undertrack. Notice the prominent circles of deformation surrounding the undertrack. Knife handle $=10 \mathrm{~cm} . C$, Deep undertracks where the tridactyl shape of the true track is unrecognizable; the only evidence for the former presence of the track is the concentric circles developed by erosion of the undertracks. Scale bar $=10 \mathrm{~cm}$. 
nent series of oval-shaped deformed layers extends for $20-30 \mathrm{~cm}$ outward from the position of the true track (fig. 2C; Milàn and Loope 2007). At this depth of erosion, no information about the animal's foot morphology is obtainable.

\section{Dinosaur Walking Dynamics}

According to a simplified model of dinosaur walking dynamics, the period of contact between the dinosaur's foot and the substrate can be divided into three distinct phases (Thulborn and Wade 1989; Avanzini 1998). The first phase is the touchdown phase, where the foot is moved forward and emplaced onto the sediment surface. This is followed by the weight-bearing phase, as the body of the animal passes over the foot that consequently compresses and deforms the sediment. Last is the kickoff phase, when the weight of the animal is transferred to the forefoot and the digits as the body moves forward and the foot is pressed backward during the kickoff and subsequently is lifted and swung toward the next touchdown phase. As evidenced by Gatesy $(2001,2003)$ and Gatesy et al. (1999), however, the movement phases are more complex and represent a continuum of interactions between the foot and the substrate. When viewed from a ground-based reaction force perspective, the phases of contact between the animal and the substrate can still be divided into three phases, although there is a gradual shift between them. At the touchdown of the foot, the substrate exercises a backward and upward force on the animal as it decelerates. This shifts to a vertical peak magnitude force as the full weight of the animal is applied to the foot in the weight-bearing phase and shifts to a forward and upward force as the animal reaccelerates during the kickoff (Roberts and Scales 2002).

\section{Structural Analysis of the Dinosaur Undertracks}

Introduction. One of the long theropod trackways encountered at the Twentymile Wash locality can be traced for more than $30 \mathrm{~m}$ where the surface has been eroded to such a depth that all of the individual tracks are exposed as undertracks. Among these tracks, three consecutive tracks display extensional and compressional fault systems that are usually associated with tectonic activity of consolidated bedrock. The tectonic activity is encountered only along a few meters of the dinosaur trackway, and the structures are restricted to the undertracks and do not extend into the surrounding rock (fig. 3). The brittle deformation of the local tracking substrate suggests that the sands of the Entrada Sandstone had attained an early diagenetic patchy consolidation of the near surface layers. In the description of the deformation of the undertracks, we use structural terms in general use adopted from the regional deformation of the upper crust (see, e.g., van der Pluijm and Marshak 2004). The tectonic impact on the theropod undertracks is outlined as subvertical planar and curved fault systems separated by low-angle thrust faults (fig. 4). In addition, minor imbricate fan thrust systems may be encountered at the rear end of the undertracks. An impact that resulted in a fold interference pattern has also been observed.

Structural Units and Associated Structures of the Undertracks. Based on the changing structural characteristics, the faulted undertracks have been subdivided into three structural units: unit A toward the front; unit $\mathrm{B}$ at the center, along the sides, and rearward; and units $\mathrm{C} 1$ and $\mathrm{C} 2$ along the front and rear margins of the undertrack (fig. 4). The structural units are separated by an extensional fault, Fx (figs. 4, 5), in the front and by low-angle thrust faults, T1-T3. Not all the structural units are represented in all three of the analyzed undertracks.

Structural unit A in the central forepart of the undertrack is outlined by a faulted structural depression (fig. 4A, 4B). The depression may be asymmetrical, with the center shifted toward the front of the track (fig. 4C, 4D). The "front" of the depression is separated from the marginal regime at the front (C1) by an extensional, backward-dipping fault (fig. 4). The asymmetrical depressions in particular are faulted along steep planar to curved faults radiating out from the depression center (fig. $4 C, 4 D$ ); the backward-directed faults are planar, while the radiating faults toward the lateral parts of the depression display an increased curvature. The identified offsets to the left (looking toward the front of the footprint) are right-lateral, while the offsets to the right are left-lateral. To the rear, the depression is cut out by a backward-directed thrust (T1) dipping toward the front below the depression. Unit A is thus lying above the Fx extensional fault in the front as well as the T1 thrust at the rear end of the structural depression (fig. $5 B$ ).

Structural unit B in the center and toward the rear may be subdivided into three segments. Segment B1, in the median part of the undertrack, has subvertical straight faults in two directions intersecting at an acute angle of ca. $50^{\circ}$, forming conjugate fault systems (fig. 4). The median segment, $\mathrm{B} 1$, is separated from the lateral segments, B2 and B3, by fault zones (FZ in fig. 5) composed of steep faults that parallel the long axis of the undertracks. 

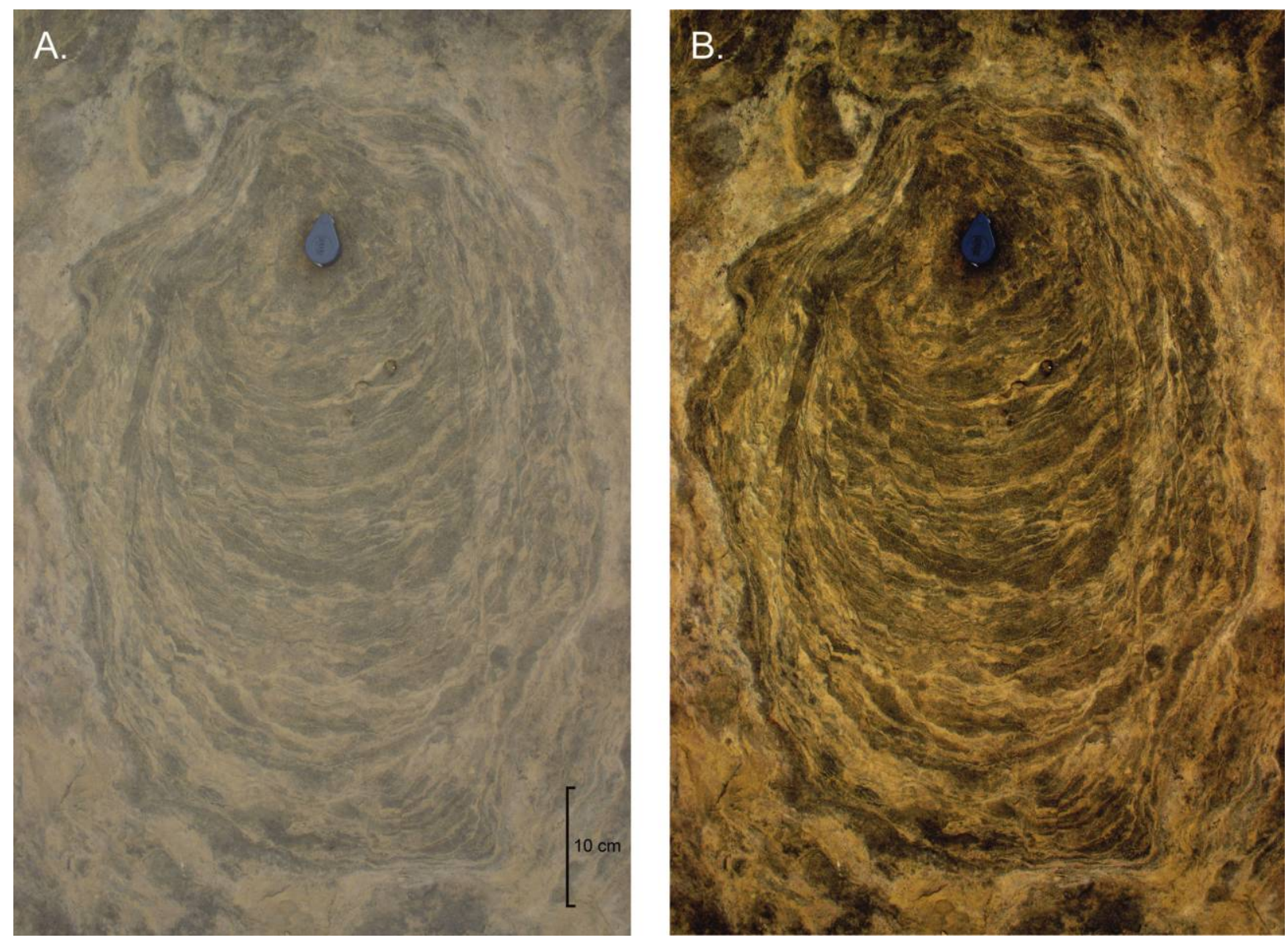

Figure 3. $A$, Photo of dinosaur undertrack with structural offsets of the thinly laminated sediment package. $B$, Same as $A$, with digital manipulation of colors and contrasts to enhance the structural deformation of the undertrack.

The lateral segments are identified by splay faults that diverge outward and backward from the parallel fault zones along B1. The offsets in the left segment (looking toward the front of the footprint) are right-lateral, while the offsets in the right segment are left-lateral (figs. 4, 5A). At the rear end, structural unit $B$ may be cut out by a low-angle thrust fault, T3, dipping toward the front below unit B (fig. 4B, $4 D$; fig. 5). Additional thrust faults, $\mathrm{T} 2$, may also lie within the B1 segment (fig. $4 B$, $4 C)$.

Structural unit $\mathrm{C}$ is encountered between the marginal fault zones, Fx and T3, and the border of the undertrack; the deformation of the undertracks dies out toward the border in the marginal regime. The most conspicuous structures of the border zone are imbricate fault systems that may occur along the front and at the rear end of the undertracks. The frontal margin may be cut up into an extensional fault system developed in the footwall block below Fx in structural regime $\mathrm{C} 1$ (figs. $4 C$, 5), while the imbricate fan at the rear end is developed in regime $\mathrm{C} 2$ in the footwall block below T3 (figs. 4B, 5).

Prototype of Faulted Undertrack in Thin-Bedded Sandsheet Deposits. The number of structural elements and composite structural systems identified in the analyzed undertracks varies from one undertrack to another. However, there is a common outline of the structural units developed, and most structural elements are shared by at least two of the three described undertracks. The similarities between the structural frameworks of the undertracks enable the construction of a schematic prototype of a faulted undertrack valid for thin-bedded sandsheet deposits (fig. 5A).

In the previous section, the structural units were described as individual composite units. There is, however, a structural coupling between the units back through the undertrack. The structural inter- 


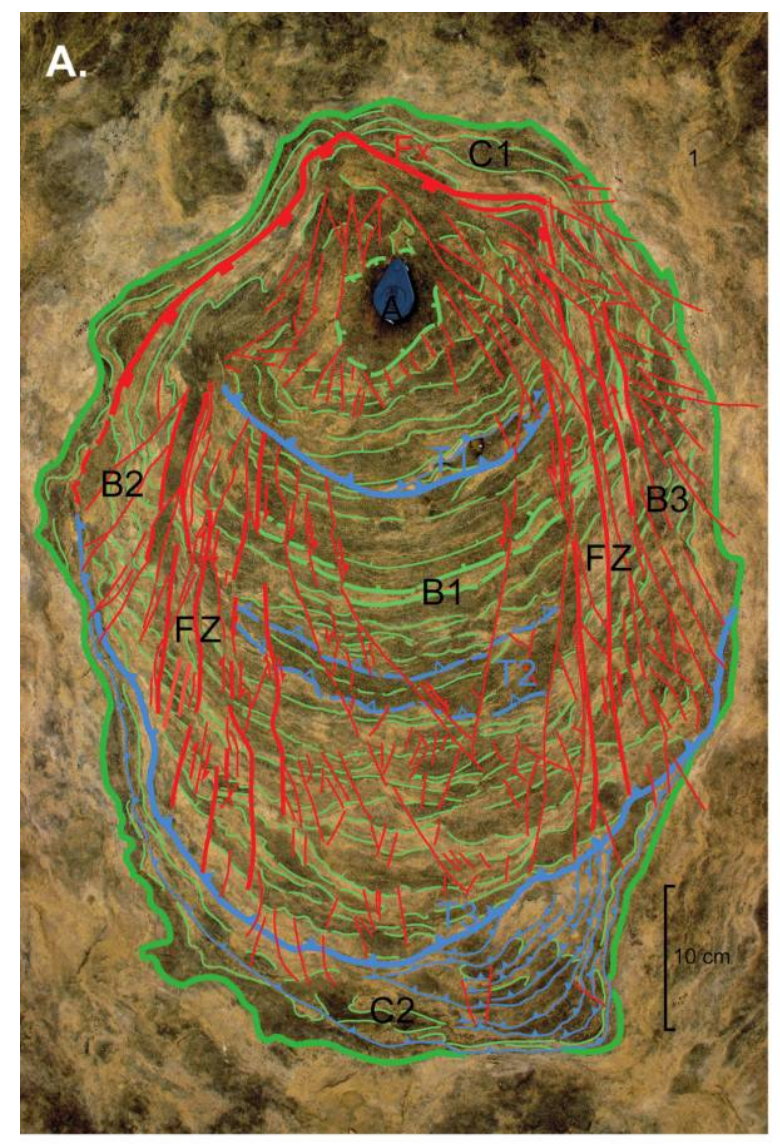

B.

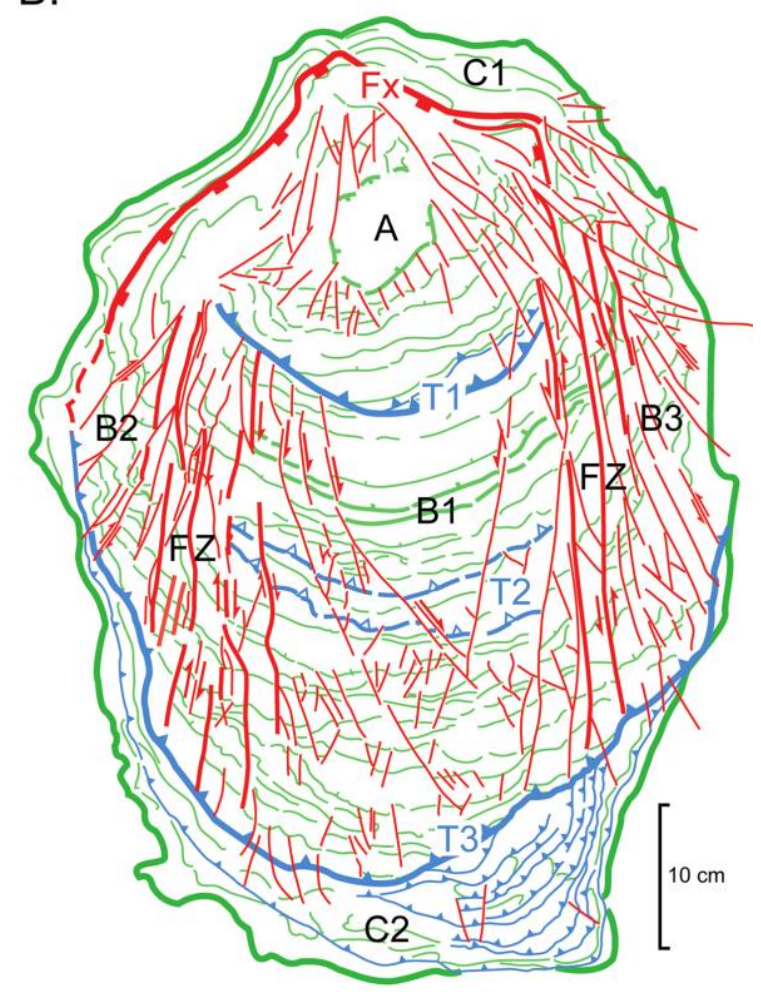

C.

D.
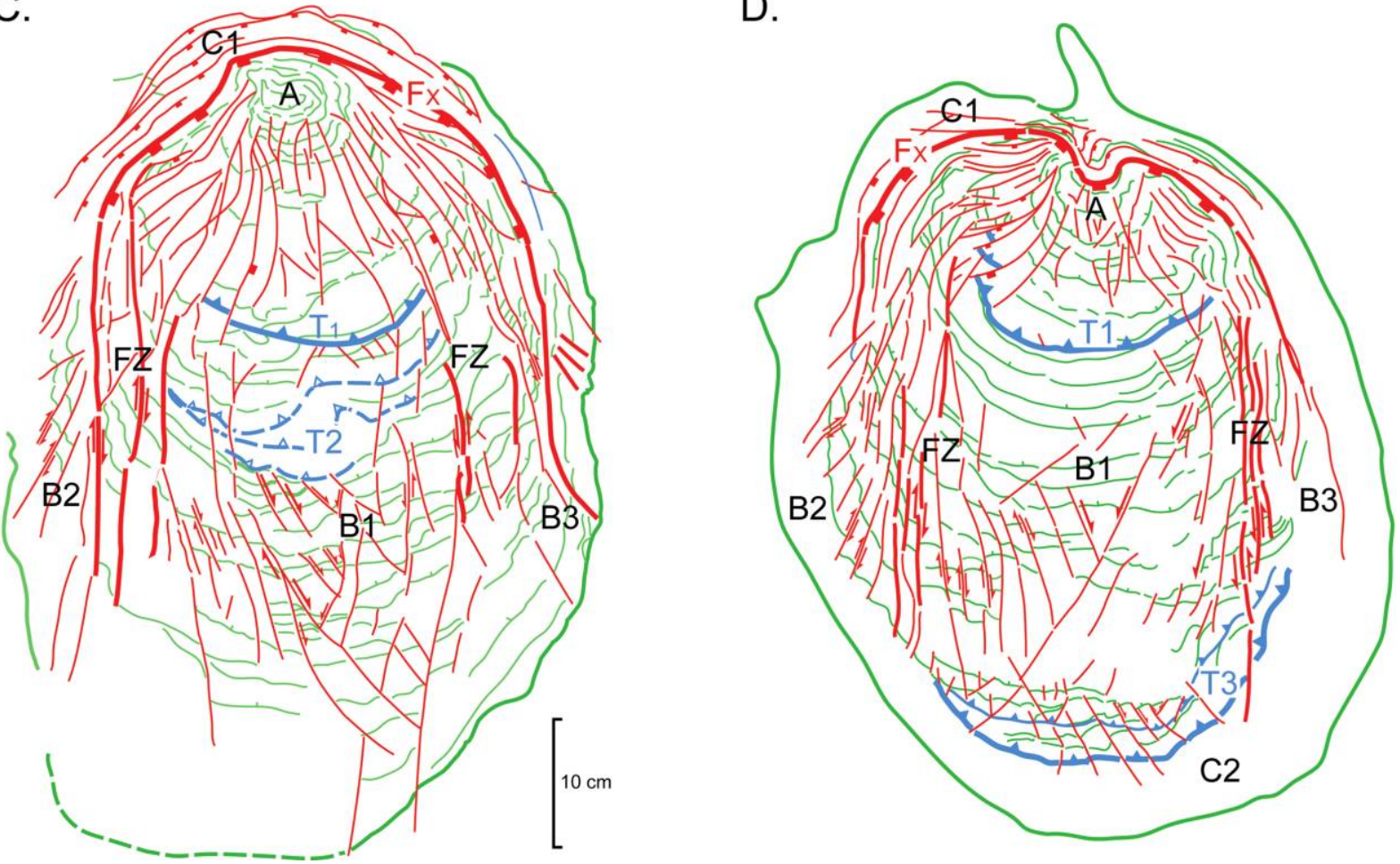
relationship is established at structural anomalies encountered along the boundaries between the structural units. In unit A there are lateral overlaps between the frontal extensional fault $(\mathrm{Fx})$ and the following more narrow intermediate thrust (T1) at the rear end of unit A (fig. 4). The overlap zones in unit A coincide with the front positions of the two lateral fault zones in unit B. The lateral fault zones are, to a great extent, restricted to unit B. In one of the footprints (fig. 4D), the rear ends of the fault zones concur with the lateral positions of the tailing thrust zone (T3; fig. 5A). The parallel fault zones may thus form a link from unit $\mathrm{A}$ in the front through unit $\mathrm{B}$ and back to unit $\mathrm{C} 2$ at the rear end of the undertrack. The geometrical relationship suggests that there may be a dynamic coupling between the structural units.

\section{Kinematic Analysis}

The structural deformation is restricted to the dinosaur undertracks in an isolated tectonic environment. The undeformed area surrounding each track is thus taken as a stationary reference region relative to the deformation identified within the undertrack. The right-lateral offsets identified in the left part of the undertrack (viewed looking toward the front) and the left-lateral offsets to the right indicate that there is an overall backward transport increasing from the margins of the undertrack and toward the median segments (fig. 5A).

The frontal extensional fault $(\mathrm{Fx})$ separates structural unit $\mathrm{A}$ in the hanging-wall block from unit $\mathrm{C} 1$ in the footwall block; the fault is upward and backward concave and parallels the front border of the undertrack (fig. 5A). As the deformation dies out toward the margin of the undertrack in the footwall block (C1), the fault movement between units $\mathrm{A}$ and $\mathrm{C} 1$ is interpreted as the result of extension and backward movement of unit A, i.e., the hanging-wall block (fig. $5 B$ ). The $\mathrm{T} 1$ thrust at the rear end of unit $\mathrm{A}$ is forward and upward concave, and unit $\mathrm{A}$ also lies above the $\mathrm{T} 1$ thrust in the hangingwall block. The offsets along the radial extensional faults in unit A indicate a downward movement toward the median segment (section line A in fig. $5 A)$. The $\mathrm{T} 1$ thrust plane may be offset along the radial faults; this is interpreted as indicating that the downward- and backward-directed movement of unit A outlasted the initial thrusting above a wider curved ramp with backward transport and uplift above both the frontal and the oblique segments of the thrust ramp. The interpreted movements along Fx and $\mathrm{T} 1$ associated with unit $\mathrm{A}$ are both oriented toward the rear end of the undertrack, and the two bounding faults must belong to a common stress regime that linked $\mathrm{Fx}$ and $\mathrm{T} 1$ together below the structural depression (unit A; fig. 5B). The T3 thrust plane at the rear end of the undertrack is forward concave and is (sub-)parallel with the boundary of the undertrack at the back. Unit B1 sits above the T3 thrust plane in the hangingwall block, while the imbricate fan thrust complex in unit C2 is developed in the footwall block. The offsets in the lateral fault zones and in the subunits B2 and B3 indicate a backward thrusting of unit B above unit C2 (fig. 5). This is in accordance with the discordant cutoff of the $\mathrm{C} 2$ fan complex in the footwall block below T3. The thrusting along T1 and $\mathrm{T} 3$ are both toward the back of the undertrack and developed in a backward-directed stress regime.

The orientation of the stress axes in unit $\mathrm{A}$ and subunit B1 can be evaluated from the fault systems in the two units. In unit $\mathrm{A}$, the extensional fault $(\mathrm{Fx})$ at the front and the radial faults within unit $\mathrm{A}$ indicate a downward movement with an initial (sub-)vertical maximum stress axis $(\sigma 1$; section line A in fig. 5). However, the movement along the $\mathrm{T} 1$ low-angle thrust fault at the rear end of unit A points to a stress field with a (sub-)horizontal maximum stress axis $(\sigma 1)$ that paralleled the long axis of the undertrack. The correlation of $\mathrm{Fx}$ and $\mathrm{T} 1$ below unit A into a common upward-concave fault system suggests that an original downward-oriented maximum stress axis was rotated toward a backward-directed maximum stress axis during rotation of unit A. In subunit B1, the intersection of the steep fault zones of the conjugate fault system defines the intermediate stress axis $(\sigma 2)$ as a (sub-)vertical-oriented axis. The maximum $(\sigma 1)$ and minimum ( 03 ) stress axes are (sub-)horizontal and bisect the acute and obtuse angles, respectively (fig. $5 A$ ). The fault zones along the lateral boundaries

Figure 4. Three dinosaur tracks showing structural deformation. A, Digitally manipulated photo of undertrack (fig. $3 B$ ) with structural interpretation. $B$, Line drawing of structural interpretation extracted from $A$. $C, D$, Line drawings of structural interpretations of two additional undertracks from the same trackway, also based on manipulated photos (not shown). See figure 5 for key to symbols and abbreviations. 

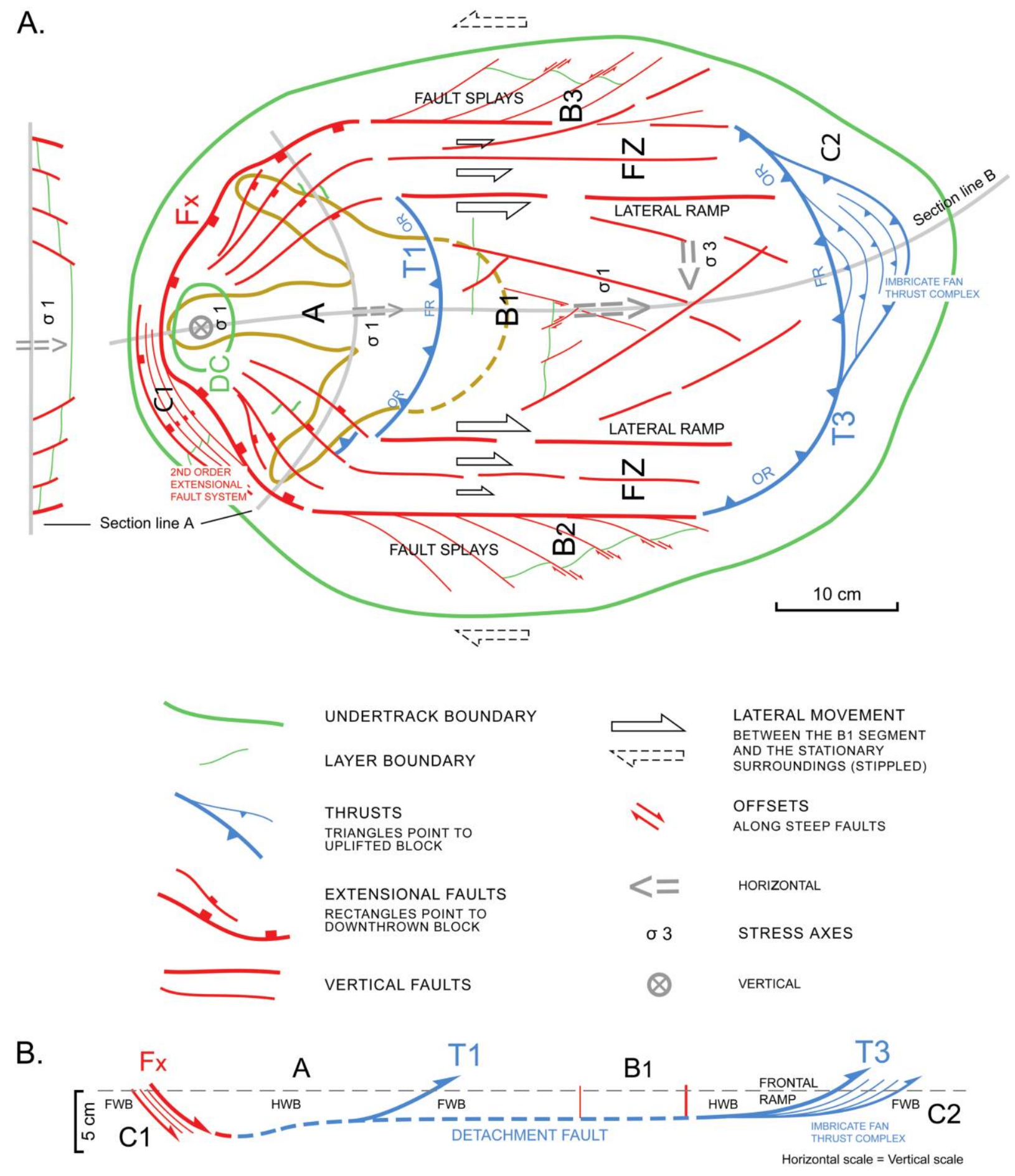

Figure 5. A, Schematic prototype undertrack based on the structural interpretations of the undertracks in figure 4 . The interpreted dimension of the original foot size is shown in orange. $A=$ frontal disk; $B 1=$ median segment; $B 2$, $B 3$ = lateral segments; $C 1=$ second-order extensional fault system; $C 2=$ imbricate fan thrust complex; $D C=$ depression center; $F R=$ frontal ramp; $F x=$ extensional fault; $F Z=$ vertical fault zone; $O R=$ oblique ramp; $T 1$, $T 3$ = thrust faults. $B$, Cross section interpreted along section line $B$ in $A$. $H W B=$ hanging-wall block; $F W B=$ footwall block. The dashed gray line indicates the current erosion level. 
of subunit $\mathrm{B} 1$ thus parallel the maximum stress axis of the central segment. Subunit B1 is a relatively stable block; no faults within the conjugate fault system cut across the entire unit. The movement within the lateral fault zones and the subunits B2 and B3 along the margins thus took place between the boundaries of the median segment (B1) and the stable area surrounding the undertrack (fig. $5 \mathrm{~A}$ ).

The movements of the subunit B1 block (toward the back of the undertrack) and the associated faults are linked in a composite thrust fault system. In map view, the bounding fault ramp is composed of the combined frontal and oblique ramps established along the $\mathrm{T} 3$ thrust, while lateral ramps are established along the parallel fault zones (fig. 5A). The subunit B1 hanging-wall block is interpreted as being separated from the stable footwall block by a detachment fault at the base (fig. $5 B$ ). The forward movement in the area toward the back of the undertrack of the imbricate fan $(\mathrm{C} 2)$ in front of $\mathrm{T} 3$ adds naturally to the B1 thrust complex in the vaning deformation front along the rear margin of the undertrack. In the opposite direction, the B1 thrust fault system can be extended back against the movement direction into unit A situated in the hanging-wall block above $\mathrm{T} 1$ at the rear end of the B1 block (relative to the movement direction of the thrust fault system; fig. 5). The T1 thrust acted as a combined frontal and oblique ramp relative to unit A, while the "lateral" ramps may be outlined by the bounding radial faults converging toward the depression center. The movement of the combined thrust fault system outlined by units A-B1-C2 can thus be traced back to a punctiform force situated at the depression center in unit A (fig. 5).

\section{Dynamic Interpretation}

Based on the structural and kinematic analyses and the reconstructed prototype of a faulted undertrack, it is possible to establish three phases of deformation related to the dinosaur foot movement. The identified deformation phases were governed by significant shifts in the dinosaur weight distribution exercised on each foot during the stride. We have integrated a sequential reconstruction of the structural deformation sequence with an interpretation of the dinosaur foot impact and the changing ground contact associated with the stacked deformation phases (fig. 6). Both the extensional fault $(\mathrm{Fx})$ and the compressional thrust faults (T1-T3) indicate a backward movement of the mapped units. The deformation is associated with an initially vertical maximum stress axis at the front of the undertrack and a horizontal maximum stress axis that parallels the long axis of the rear part of the undertrack (fig. 5). There is thus no indication of a decelerating touchdown phase with a forwarddirected impact of the dinosaur foot at the level of observation $(\sim 10 \mathrm{~cm}$ below the original tracking surface). Our description and interpretation of the dynamic phases is based on a two-dimensional sequential reconstruction along the center line of the prototype undertrack (figs. 5, 6).

1 . The starting point for the structural reconstruction is illustrated by the dinosaur foot impact into the ground; the maximum stress axis was vertical and directed downward (fig. 6A). The maximum vertical compression occurred when the full weight of the animal was applied to the ground during the weight-bearing phase. The layers below the footprint were compressed, and the undertrack formed a structural depression (not illustrated in fig. 6). Additional sideways pressure caused the formation of a raised rim of displaced material around the foot.

2. The early deformation of the undertrack formed in phase 1 formed a composite disk comprising structural unit A (figs. 5, 6B). The disk developed as a hanging-wall block that rotated above a curved fault. The fault surface is composed of the extensional fault $(\mathrm{Fx})$ at the front and the thrust fault (T1) at the rear end of unit A. The rotation and backward movement of the disk was associated with rotation of the principal stress axes; the vertical stress component was reduced while a backward-directed stress component started to grow (fig. $6 B$ ). The initial disk rotation followed the weight-bearing phase (1); the weight of the dinosaur was gradually transferred from the rear part of the foot toward the front end of the digits during the early kickoff phase. The metatarsal pad was lifted clear of the ground during this stage (2) as the tips of the digits were forced downward. The shift in weight distribution formed a rotational disk that cut through to the original ground surface behind the foot.

3. During the intermediate and late kickoff phase, deformation extended backward, as evidenced by the development of new thrust ramps, i.e., an intermediate ramp T2 (figs. $4 A-4 C, 6 C$ ), and/or the T3 thrust ramp at the rear end of the undertrack (fig. $4 A, 4 B, 4 D$; fig. $6 D$ ). The backward extension of the deformation accompanied a growing horizontal stress component at the expense of the vertical stress component. This indicates a continued rotation of the principal stress axes exerted by the dinosaur foot at the frontal disk. The continued rotation of the front disk is interpreted as being governed by the ongoing rotation of the dinosaur foot during performance of the kickoff phase. Along with the rotation, the rear part of the dinosaur foot was lifted off the 

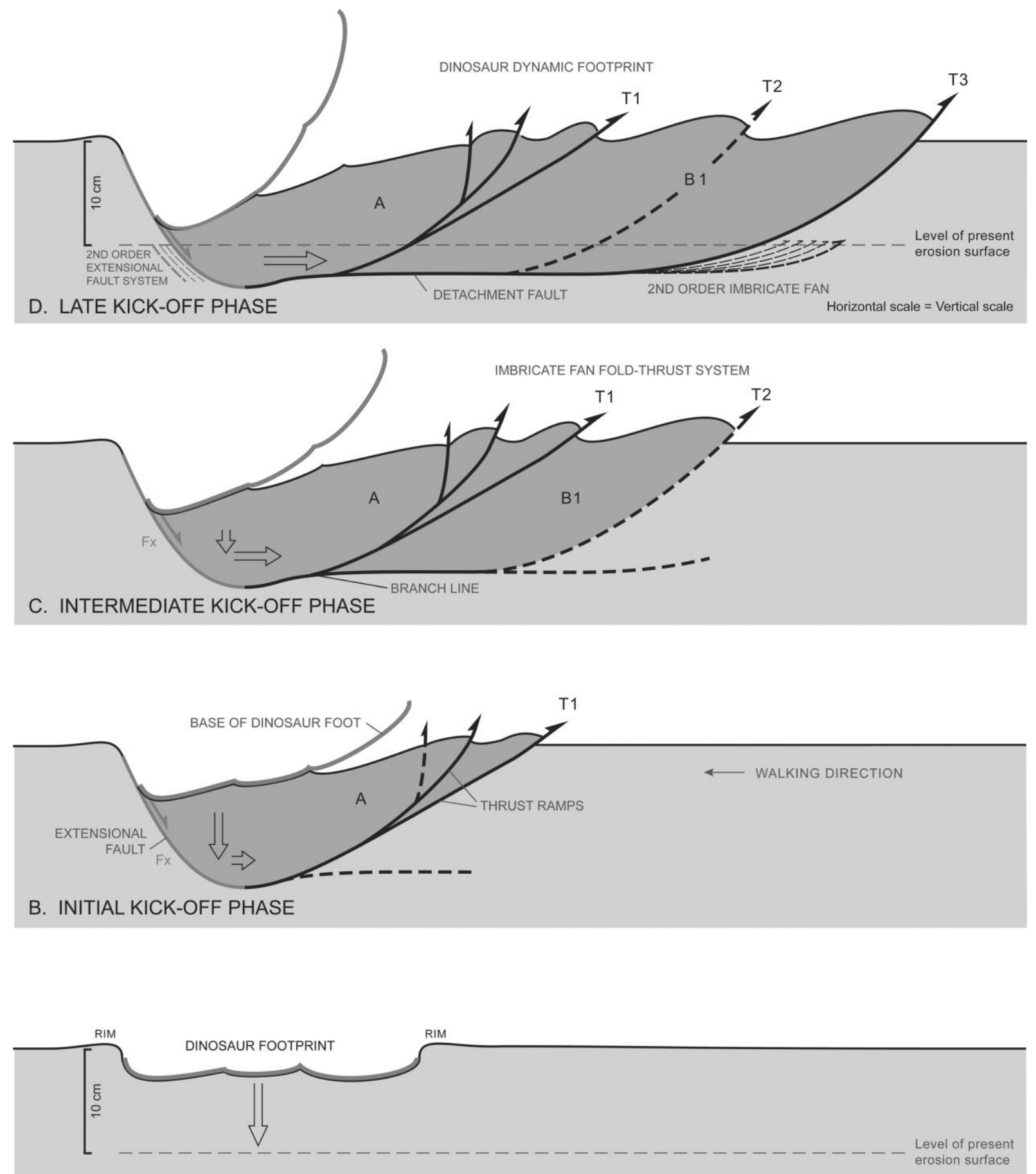

Horizontal scale $=$ Vertical scale

Figure 6. Structural interpretation of the dynamic evolution of dinosaur foot impact during the weight-bearing and kickoff phases. A, Dinosaur footprint and rim deformation established during the peak weight-bearing phase. $B$, Formation of the frontal disk $(A)$ during the initial kickoff phase. $C$, Continued rotation and forward movement of the dinosaur foot contact during the intermediate kickoff phase lead to a growing backward-directed movement vector and the evolution of an imbricate fan thrust system. $D$, During the late kickoff phase, the increased horizontal vector was responsible for the continued backward extension of the fold-thrust system that faded out at the sole in the second-order imbricate fan at the back. 
ground, and the backward-directed power of the foot was further concentrated on the digits (fig. 6C, 6D).

The lateral overlap between the broad frontal extensional fault $(\mathrm{Fx})$ and the more narrow thrust fault (T1) at the rear end of the frontal disk (fig. 5) shows that the backward-directed power was concentrated toward the center during the rotation of the disk. This interpretation is further supported by the normal faulting and offset of the thrust ramp (T1) along the radiating faults, as illustrated by the map of the undertrack and the cross section along line $A$ (fig. 5). The structural evolution shows that the backward-directed force of the dinosaur was transferred to the middle digit during the late kickoff phase. Furthermore, the structures suggest that the lateral digits did not move toward the middle digit; if this had been the case, the lateral digits would have left a transverse impact structure interfering with the radial faults extending from the frontal depression center, and this did not happen (figs. 4, 5).

The backward translation of the force from the frontal disk was transferred along the two lateral fault zones combined with a basal detachment fault (fig. 5). The similar widths of the frontal extensional fault $(\mathrm{Fx})$ and the thrust fault to the rear (T3) correlate with the outer margins of the lateral fault zones (figs. 4, 5). This may indicate that the initial backward movement took place across a deformation zone that was governed by the width of the dinosaur foot during the maximum weight-bearing phase. The subsequent narrowing of the backward translation force during the kickoff phase established above may also be inferred from the increased movement across the lateral fault zones toward the median segment (B1) because the width of the median segment correlates with the width of the T1 thrust fault (figs. 4, 5).

\section{Discussion}

Structural Deformation Sequence of Undertracks. The structural deformation analysis of the dinosaur undertracks has revealed a set of minor-scale structures that are analogous to crustal-scale orogenic structures usually associated with plate tectonics. The thrust ramp systems mapped from the dinosaur undertracks mimic fold-thrust structures from foreland belts known from the Canadian Rocky Mountains and the Appalachians. The deformation is restricted to individual undertracks, and each dinosaur step developed its own tectonic microcosm during the contact with the substrate. The development of recognizable structures during the dinosaur stride depended on the rheological char- acteristics of the substrate and the presence of structural markers, e.g., laminations. The size of the structures associated with the undertracks may be compared to laboratory experiments performed during sandbox analogue models of fault-related folding (Storti et al. 1997).

The development of the undertrack structures analyzed in this article requires special strength and competency conditions that are usually not encountered in unconsolidated sediments close to the earth's surface. The yield strength of unconsolidated, grain-supported clastic sediments is close to zero at the earth's surface, and this may be the reason why undertrack structures similar to the structures analyzed in this article have not been described before. In order for us to establish the strength and competency necessary for the development of the observed structures, the sands of the Entrada Sandstone locally must have attained an early diagenetic consolidation of the near surface layers. This may have been accomplished by early, near-surface cementation, driven by evaporation during the dry season under a subtropical, monsoon climate (Loope and Rowe 2003).

In the trace fossils encountered today, it is not possible to measure the rheological properties of the substrate during dinosaur track making. Furthermore, rheological properties also depend on the strain rate, i.e., the elongation per unit time. The duration of the isolated tectonic microcosm created during each dinosaur step lasted only a few seconds. Even compared to the small size of the analyzed structures, the growth and release of the established stress were extremely rapid. The strain rates of the structures developed in the undertracks were several orders higher than the strain rates encountered during orogenic deformation in a plate tectonic setting in the earth's crust. The high strain rate established during each dinosaur step may thus have increased the relative competence of the walking substrate. Based on the contemporaneous development of faults and folds in the fold-thrust structures of the undertracks, it is estimated that the rheological conditions illustrate a system close to the brittle-ductile transition at the time of deformation.

Tracking and Disk Formation. The well-developed disks, bound by thrust ramps described from this study, are the result of an initial powerful downward movement of the foot, followed by an almost entirely backward-directed movement. The tracks were emplaced on a horizontal surface, and the backward-directed deformations resulted from the powerful backward foot movements during the stride. Disks of displaced sediment associated with 

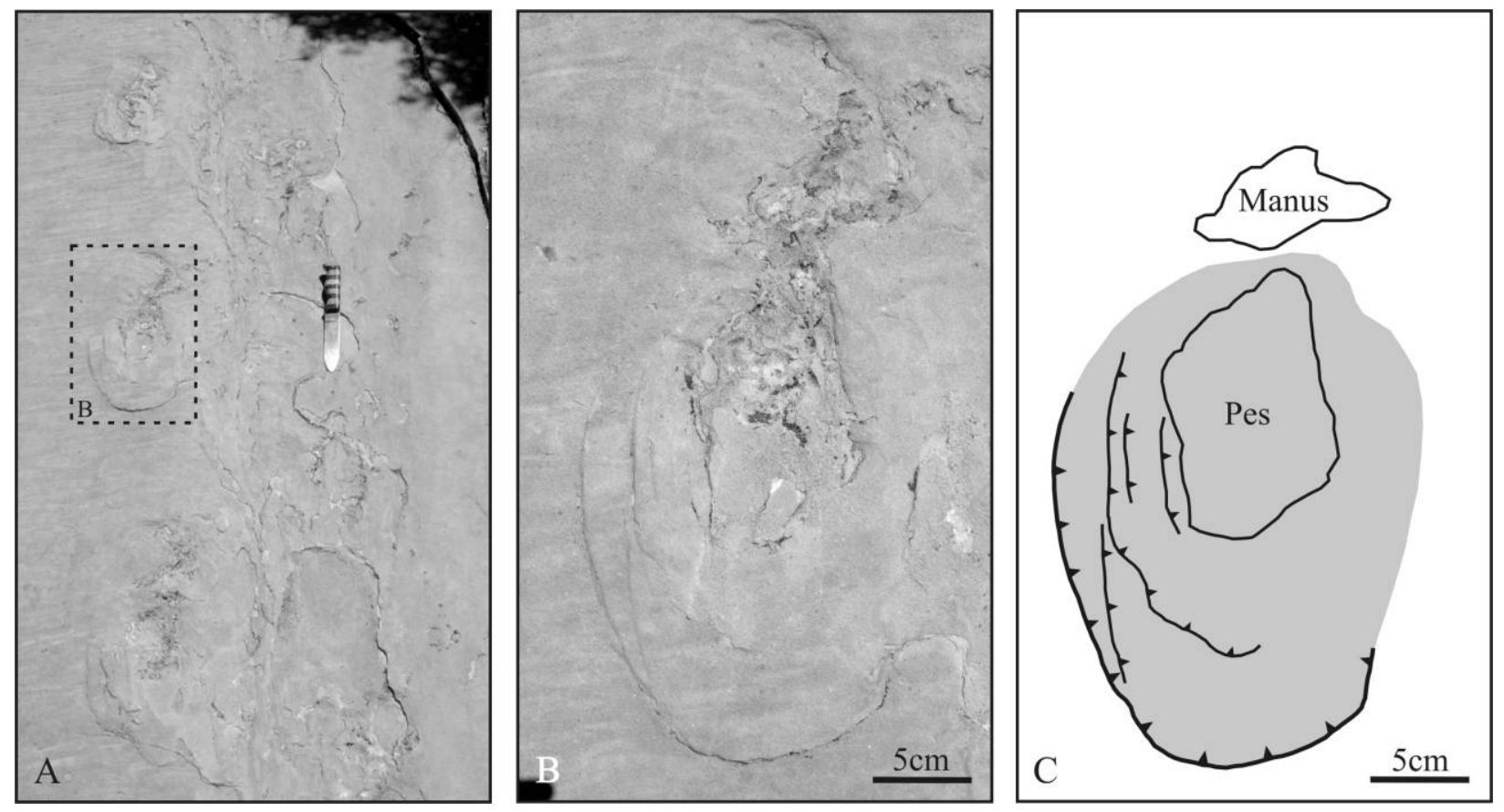

Figure 7. Trackway from a large reptile walking up the slope front of a paleodune in the Permian Coconino Sandstone of Grand Canyon exposed along the Hermit Trail at the South Rim of Grand Canyon. A, Trackway consisting of paired manus-pes imprints and a sinusoidal tail drag mark. Rectangle indicates location of close-up shown in $B$ and $C$. $B$, Close-up photo of manus and pes couple, where large disks of displaced material are evident on the tracking surface behind the tracks. $C$, Interpretative drawing of the structures evident on the tracking surface. The disk is composed of a number of faults, most prominently developed on the outside of the trackway. The shaded area indicates the total area of sediments disturbed by the foot.

tracks of dinosaurs and other vertebrates have hitherto received little attention in vertebrate ichnology. However, a few studies describe similar structures, most of them from tracks and trackways in eolian settings (Lockley 1991). Such disks of displaced sediment are especially abundant in the numerous reptilian tracks and trackways from the Coconino Sandstone of Grand Canyon (McKee 1944), and Pleistocene tracks and trackways from now extinct goats from Mallorca walking up the slopes of ancient eolianites have preserved similar structures. These tracks have preserved well-developed disks of displaced sediment on the downslope side of the tracks (Fornós et al. 2002). A similar disk of displaced material is reported from the downslope side of a hoof imprint in a Quaternary eolianite from the Greek island of Rhodes (Milàn et al., forthcoming). Indirect evidence of disk formation in connection with tracks comes from eroded undertracks of humans and cattle in Roman-age beach rock from Rhodes, where the different grain sizes of the beach rock are arranged in semilunate units on the downslope side of the tracks (Bromley et al., forth- coming). Permian reptile tracks from the eolian Coconino sandstone exposed along the Hermit Trail at the South Rim of Grand Canyon further show large disks of displaced material behind each footprint in a trackway progressing up the slope front of a paleodune (McKee 1944; fig. 7).

The formation of the disks described in the examples above is not directly comparable to that of the disks described from the dinosaur tracks in this article. The identification of backward force exercised on the tracking substrate by the Permian reptiles (McKee 1944), the humans and animals from Rhodes (Bromley et al., forthcoming; Milàn et al., forthcoming), and the Pleistocene goats (Fornós et al. 2002) was supported by the fact that these animals were walking on sloping surfaces, so that the impact angle of the foot exercised a stronger force onto the substrate, thus enhancing the formation of displaced disks behind the animals. This is in contrast to the tracks of this article, which were emplaced on a horizontal surface, and which can only be the result of a powerful backward component of the foot movement during the stride. 
Reconstruction of Walking Dynamics. Even though the true tracks are eroded away, based on the structural and kinematic analyses of the undertracks from the Twentymile Wash locality, it is possible to establish the walking dynamics of the actual dinosaur responsible for the studied (under-)trackway. This study thereby differs from previous reconstructions of dinosaur walking dynamics that were based on footprints; the previous analyses used the direct deformation of the true track to reconstruct the foot movements during the stride.

The complex system of displaced disks from the tracks in this study reveals information about the end of the weight-bearing phase and the kickoff phase, while no remains of deformation from the touchdown phase are present at this level of erosion. Based on the described deformations, the following reconstruction of the walking dynamics can be established. During the end of the weight-bearing phase, the weight of the dinosaur was transferred toward the distal anterior parts of the foot, which then became more deeply imprinted into the sediment (fig. 6B), as was almost always the case with tridactyl theropod dinosaurs. As the body of the animal moved forward in the initial part of the kickoff, the weight was lifted clear of the rear end of the foot and the weight of the animal became concentrated on the digits. As the dinosaur accelerated forward in the kickoff, the foot was forced backward, with all the weight concentrated on the distal ends of the digits (fig. 6C, 6D). This implies that the initial imprint and associated undertracks of the anterior end of the foot, which reflect a downward movement, was subsequently deformed by the posterior movements of the sediment caused by the foot itself.

\section{Conclusions}

Deformed dinosaur undertracks from the Entrada Sandstone exhibit a complex set of local thrust ramp systems created in isolated tectonic systems during contact of dinosaur feet with the ground. The small-scale, dinosaur-induced thrust ramp systems are similar to crustal-scale tectonics encountered in orogenic foreland basins.

During dinosaur track making, a rotated disk was created below the dinosaur foot during the transition from the weight-bearing phase to the kickoff. Subsequently, during the kickoff phase, the disk was forced backward during the creation of the imbricate fold-thrust system.

The deformation of the tracking surface suggests that the Entrada Sandstone locally had attained an early diagenetic consolidation, facilitating the development of the brittle-ductile structures. The tectonic deformation induced by the dinosaur's foot created a disturbed zone of up to three times the length of the dinosaur foot. The incorporation of structural analysis into ichnological work has proven to be useful in the reconstruction and understanding of dinosaur track making.

\section{R E F E R E N C E S C I T E D}

Allen, J. R. L. 1989. Fossil vertebrate tracks and indenter mechanics. J. Geol. Soc. Lond. 146:600-602.

. 1997. Subfossil mammalian tracks (Flandrian) in the Severn Estuary, S.W. Britain: mechanics of formation, preservation and distribution. Philos. Trans. R. Soc. B 352:481-518.

Avanzini, M. 1998. Anatomy of a footprint: bioturbation as a key to understanding dinosaur walk dynamics. Ichnos 6:129-139.

Breithaupt, B. H.; Matthews, N. A.; and Noble, T. A. 2004. An integrated approach to three-dimensional data collection at dinosaur tracksites in the Rocky Mountain West. Ichnos 11:11-26.

Bromley, R. G.; Uchman, A.; Milàn, J; and Hansen, K. S. Forthcoming. Rheotactic Macaronichnus, and human and cattle trackways in Holocene beachrock, Greece: reconstruction of palaeoshoreline orientation. Ichnos.

Brown, T., Jr. 1999. The science and art of tracking. New York, Berkley, 219 p.

Fornós, J. J.; Bromley, R. G.; Clemmensen, L. B; and Rodriguez-Perea, A. 2002. Tracks and trackways of $M y-$ otragus balearicus Bate (Artiodactyla, Caprinae) in
Pleistocene aeolianites from Mallorca (Balearic Islands, western Mediterranean). Palaeogeogr. Palaeoclimatol. Palaeoecol. 180:277-313.

Foster, J. R.; Hamblin, A. H.; and Lockley, M. G. 2000. The oldest evidence of a sauropod dinosaur in the western United States and other important vertebrate trackways from Grand Staircase-Escalante National Monument, Utah. Ichnos 7:169-181.

Gatesy, S. M. 2001. Skin impressions of Triassic theropods as records of foot movements. Bull. Mus. Comp. Zool. 156:137-149.

- 2003. Direct and indirect track features: what sediment did a dinosaur touch? Ichnos 10:91-98.

Gatesy, S. M.; Middleton, K. M.; Jenkins, F. A., Jr.; and Shubin, N. H. 1999. Three-dimensional preservation of foot movements in Triassic theropod dinosaurs. Nature 399:141-144.

Lockley, M., and Hunt, A. P. 1995. Dinosaur tracks and other fossil footprints of the western United States. New York, Columbia University Press, 338 p.

Lockley, M. G. 1991. Tracking dinosaurs. New York, Cambridge University Press, 238 p. 
1997. The paleoecological and paleoenvironmental utility of dinosaur tracks. In Farlow, J. O., and Brett-Surman, M. K., eds. The complete dinosaur. Bloomington, Indiana University Press, p. 554-578.

Loope, D. B., and Rowe, C. M. 2003. Long-lived pluvial episodes during deposition of the Navajo Sandstone. J. Geol. 111:223-232.

Loope, D. B., and Simpson, E. L. 1992. Significance of thin sets of eolian cross-strata. J. Sediment. Res. 62: 849-859

Manning, P. 2004. A new approach to the analysis and interpretation of tracks: examples from the dinosauria. In McIlroy, D., ed. The application of ichnology to palaeoenviromental and stratigraphic analysis. Geol. Soc. Lond. Spec. Publ. 228:93-123.

McKee, E. D. 1944. Tracks that go uphill. Plateau 16:6172.

Milàn, J.; Avanzini, M.; Clemmensen, L. B.; Garcia-Ramos, J. C.; and Piñuela, L. 2006. Theropod foot movement recorded from Late Triassic, Early Jurassic and Late Jurassic fossil footprints. In Harris, J. D.; Lucas, S. G.; Spielmann, J. A.; Lockley, M. G.; Milner, A. R. C.; and Kirkland, J. I., eds. The Triassic-Jurassic terrestrial transition. N. M. Mus. Nat. Hist. Sci. Bull. 37:352-364.

Milàn, J., and Bromley, R. G. 2006. True tracks, undertracks and eroded tracks, experimental work with tetrapod tracks in laboratory and field. Palaeogeogr. Palaeoclimatol. Palaeoecol. 231:253-264.

Milàn, J.; Bromley, R. G.; Titschack, J.; and Theodorou, G. Forthcoming. A diverse vertebrate ichnofauna from a Quaternary eolian oolite from Rhodes, Greece. In Bromley, R. G.; Buatois, L. A.; Márango, M. G.; Genise, J. F.; and Melchor, R. N., eds. Sediment-organism interactions: a multifaceted ichnology. SEPM Spec. Publ.

Milàn, J.; Clemmensen, L. B.; and Bonde, N. 2004. Vertical sections through dinosaur tracks (Late Triassic lake deposits, East Greenland): undertracks and other subsurface deformation structures revealed. Lethaia 37:285-296.

Milàn, J., and Loope, D. B. 2007. Preservation and erosion of theropod tracks in eolian deposits: examples from the Middle Jurassic Entrada Sandstone, Utah, U.S.A. J. Geol. 115:375-386.

Roberts, T. J., and Scales, J. A. 2002. Mechanical power output during running accelerations in wild turkeys. J. Exp. Biol. 205:1485-1494.

Storti, F.; Salvini, F.; and McClay, K. 1997. Fault-related folding in sandbox analogue models of thrust wedges. J. Struct. Geol. 19:583-602.

Thompson, A. E., and Stokes, W. L. 1970. Stratigraphy of the San Rafael Group, southwest and south central Utah. Utah Geol. Mineral. Surv. Bull. 87:1-53.

Thulborn, R. A., and Wade, M. 1989. A footprint as history of movement. In Gillette, D. D., and Lockley, M. G., eds. Dinosaur tracks and traces. New York, Cambridge University Press, p. 51-56.

van der Pluijm, B. A., and Marshak, S. 2004. Earth structure: an introduction to structural geology and tectonics. New York, Norton, 656 p. 